\title{
The nature of seismic sources associated with a proton-reach solar flare
}

\author{
Valentyna V. Zharkova ${ }^{1}$ and Serhij I. Zharkov ${ }^{2}$ \\ ${ }^{1}$ Department of Computing and Mathematics, University of Bradford, Bradford, UK, \\ email: v.v.zharkova@brad.ac.uk \\ ${ }^{2}$ Department of Applied Mathematics, University of Sheffield, Sheffield, UK \\ email: s.zharkov@sheffield.ac.uk
}

\begin{abstract}
The momenta and start times measured from the TD diagrams in 3 seismic sources observed in the flare of 28 October 2003 are compared with those delivered to the photosphere by different kinds of high energy particles as well as by the hydrodynamic shocks caused by these particles. The energetic protons with energy power laws combined with quasi-thermal ones are shown to form hydrodynamic shocks deeply in a flaring atmosphere which deliver the required momentum to the photosphere within a measured timescale. The seismic waves observed in two sources associated with $\gamma$-rays can be explained by the momenta produced by hydrodynamic shocks caused by mixed proton beams and jets. The seismic wave in the source asociated with HXR only and delayed by 4 and 2 minutes from the first and second HXR bursts is likely to be associated with a hydrodynamic shock occurring from precipitation of a very powerful and hard electron beam possibly mixed with quasi-thermal lower energy protons.
\end{abstract}

Keywords. Sun: activity, Sun: sunspots, Sun: interior, Sun: magnetic field, methods: data analysis, methods: statistical.

\section{Introduction}

A comparison of the first observations of a solar quake (Kosovichev and Zharkova, 1998) with the theoretical model of a sesimic repsonse (Kosovichev and Zharkova, 1995) revealed that the momentum required to produce the observed seismic response $\left(\sim 2 \cdot 10^{22}\right.$ $\left.\mathrm{g} \mathrm{cm} \mathrm{s}^{-1}\right)$ is one order of magnitude higher than those of $\sim 10^{21} \mathrm{gcm} \mathrm{s}^{-1}$ observed from the plasma downflows in the MDI dopplergrams. Also the travel time of this shock to the photosphere is more than 2 minutes while the time, at which the helioseismic response started in TD diagrams, coincides very closely with the time of the hard X-ray impulse. Hence, there should be some additional sources that can deliver the required momentum to the solar photosphere within a very short timescale coinciding with the start time of a hard X-ray impulse.

Recent observations reported helioseismic emission from the solar flares of 2003 October 28 and 29 using the helioseismic holography technique (Donea and Lindsey, 2005; DL05 thereafter), with another 5 sources occurred in the active region NOAA 10486 with the seismic emission at frequencies from $3 \mathrm{mHz}$ to $7 \mathrm{mHz}, 4$ of them in the flare 28 October 2003 (DL05). In order to establish a connection between high energy particles and the seismic source agents, in the present paper we study the velocities of vertical and horizontal displacements, or the ridges, associated with these seismic waves in the flare 28 October 2003, by applying the time-distance diagram technique and by deducing the momenta required to cause the observed ridges. Then we can compare them with those delivered by high energy particles and hydrodynamic shocks occurring in the chromosphere in response to the particle injections. 


\section{Description of the observations}

The X17.2 flare occurred on the 28th October 2003 in the very active region NOAA 10486 at the location 18E20S. It started as observed by GOES from 9:41 UT lasting until 11:24 UT with the maximum at 11:10 UT in soft X-rays and until 18:00 UT in $H_{\alpha}$ emission.

In the first phase there were $2 \gamma$-continuum peaks produced mainly by a bremsstrahlung spectrum generated by electrons with energies up to $150 \mathrm{MeV}$ with a small $\gamma$-ray increase in the range of 1.5-7 MeV (Gross et al., 2004, Kuznetsov et al., 2006). In this phase the $\gamma$ line emission was also observed by the KORONAS that indicates a presence of protons with the energies $>30 \mathrm{MeV}$ but not higher than $200 \mathrm{MeV}$ because of the absence of photons from the $\pi$-decay process. The photon energy spectra obtained during the first phase are single power laws with the spectral indices about 2 at the lower energy part $(<70 \mathrm{KeV})$ and 3.5-4 at higher energies Kuznetsov et al. 2006.

The second phase, a delayed one, where the other 2 peaks are observed in $\gamma$-rays by KORONAS, INTEGRAL and RHESSI, has very noticeable plateaus in the energy spectra in the range of 25-100 MeV appearing at about 11:06:10 UT. Also there are higher energy protons $>200 \mathrm{MeV}$ appearing after 11:06:00 UT indicated by a presence of $\pi$-decay photons (Kuznetsov et al., 2006, Share et al., 2004).

\section{The observed seismic sources}

MDI dopplergrams obtained aboard SOHO from 11:00 UT until 12:00 UT with 1 minute cadence are used. These observations were supported by the MDI magnetograms and white light images taken from the Solar Feature Catalogues (SFCs) at the times closest to the flare setup time?.

For each seismic source the datacube of $120 \times 120 \mathrm{Mm}$ centred in the centre of gravity of the downward Doppler motions in this source was extracted. These were re-mapped them into the polar coordinates to which the Fourier technique is applied from the centre where the initial impulse (the center of gravity) up to $120 \mathrm{Mm}$. In order to precisely detect the seismic wave centres, we selected the areas of 20x20 pixels around the locations provided by DL05 (Table 1, in Zharkova and Zharkov, 2007) and used to define total areas for each quake and their centres of gravity.

In general, in all the MDI dopplergrams we have detected 11 locations with downward motion larger than $1 \mathrm{~km} / \mathrm{s}$ while only three of them having revealed detectable ridges, or quakes (Zharkova and Zharkov, 2007). The time-distance diagrams for the seismic sources S1-S3 reveal that the start times of the seismic waves are slightly different for each source varying from about 11:05:00-11:06:00 UT for the source S1 to 11:06:00 UT for the sources S2 and S3. These times are more than 3 minutes later than the first maximum of hard X-rays for the source S1 but close to the second maximum in both HXR and $\gamma$-rays pointing to the presence of high energy protons $(>200 \mathrm{MeV})$ for the sources S2, S3 Kuznetsov et al. 2006, Share et al 2004.

The downward velocities $V_{\text {vert }}$ directly in these 3 sources measured from the dopplergrams were $2.15 \mathrm{~km} / \mathrm{s}$ for S1, $2.0 \mathrm{~km} / \mathrm{s}$ for $\mathrm{S} 2$ and $1.75 \mathrm{~km} / \mathrm{s}$ for S3, while for another 8 of 11 sources the downward velocities were lower than $1.6 \mathrm{~km} / \mathrm{s}$. The durations of the downward motions do not exceed 1.5 -2.0 minutes, starting at or after 11:05:00 UT with the maximum a minute later and then decreasing for another 30-60 seconds back to the pre-flare magnitude. Also the initial velocity in the source S1 is higher and the seismic wave propagates towards the $120 \mathrm{Mm}$ edge of the datacube slightly faster than in other two sources S2 and S3. 


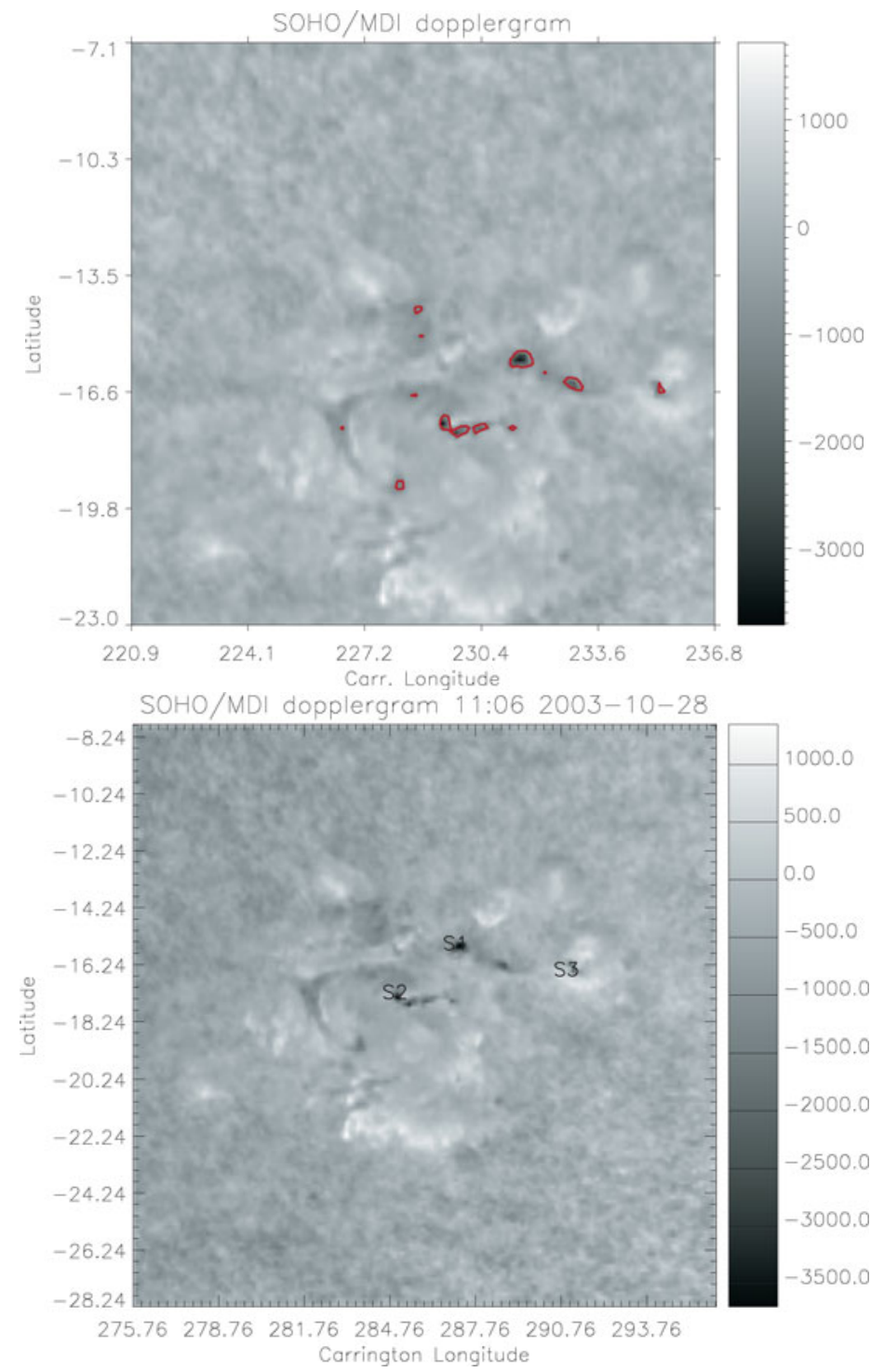

Figure 1. The locations of 11 Doppler sources (red contours) with the downward motion higher than $1 \mathrm{~km} / \mathrm{s}$ (top image) but only 3 of them with the detectable seismic responses in the locations specified in columns 1-3 of Table 1 (bottom image).

The areas of the seismic sources, defined by a presence of the downward motions and by detectable ridges, were about $5.05 \times 10^{17} \mathrm{~cm}^{2}$ for the source $\mathrm{S} 1,3.34 \times 10^{17} \mathrm{~cm}^{2}$ for the source S2 and $3.22 \times 10^{17} \mathrm{~cm}^{2}$ for the source S3. Then by using the downward velocities above and comparing with the theoretical seismic ridges (Kosovichev and Zharkova, 1995, 1998) one can deduce the momenta required to cause the observed ridges: $4.0 \times 10^{22}$ $\mathrm{g} \cdot \mathrm{cm} \cdot \mathrm{s}^{-1}(\mathrm{~S} 1), 3.7 \times 10^{22} \mathrm{~g} \cdot \mathrm{cm} \cdot \mathrm{s}^{-1}(\mathrm{~S} 2)$ and $3.1 \times 10^{22} \mathrm{~g} \cdot \mathrm{cm} \cdot \mathrm{s}^{-1}(\mathrm{~S} 3)$. 


\section{Results and discussion}

\subsection{The momenta delivered by beams and hydrodynamic shocks}

The momentum $P_{e, p}$ delivered in pure collisions by an electron or proton beam with a spectral index $\gamma_{e, p}$ and a lower energy cutoff $E_{l o w_{e, p}}$ can be evaluated as ?:

$$
P_{e, p} \simeq \sqrt{2 m_{e, p}} N_{e, p} \frac{\gamma_{e, p}}{\gamma_{e, p}+0.5} \cdot E_{l o w_{e, p}}^{-0.5} .
$$

It should be emphasized that this is the upper limit of the momentum carried downwards to the photosphere by electrons or protons since it is calculated without taking into account pitch angle scattering and wave dissipation for protons or Ohmic dissipation for electrons that can reduce its magnitude by a few factors (Zharkova and Gordovskyy, 2006)

For the known plasma mass density $\rho=m_{H} \cdot n$ where $n$ is the particle density per volume defined from hydrodynamic solutions, this momentum delivered by a hydrodynamic shock can be as follows:

$$
P_{h d}=\Sigma_{t} m v(t) \approx \rho \cdot A \cdot v^{2} \cdot \tau,
$$

where $\tau$ is a duration of the impact causing the seismic waves, $m$ is the mass of the plasma delivering the momentum related to the flaring area $A$ where the momentum is deposited, $V$ is a starting velocity at the moment of impact.

\subsection{The momenta delivered by beams}

\section{The source $S 1$}

For the flare area of about $5.05 \times 10^{17} \mathrm{~cm}^{2}$ defined by the area of a downward Doppler motion Figure 1 in the source S1, the momentum delivered to this area by the electron beam was about $2 \cdot 10^{20} \mathrm{~g} \cdot \mathrm{cm} / \mathrm{s}$. Obviously, this is not sufficient to deliver the required momentum of $(3-4) \times 10^{22}$ as reported from the TD diagram for S1 (see $\S 3$ ).

The sources $S 2$ and $S 3$.

The sources S2 and S3 appear close to the locations of hard X-ray emission and within the circle denoting $\gamma$-ray emission observed by RHESSI Hurford et al. 2006. The spectral indices of the proton energy spectra observed by INTEGRAL in the phase B after 11:06:00 UT vary from 3 to 3.8 Kiener et al. 2006 or by the RHESSI measurements vary $2.8 \mp 0.4$ with a total number of protons observed estimated at about $10^{33}$ Share et al 2004 . The lowest energy was about $30 \mathrm{MeV}$ but it can be decreased to $2 \mathrm{MeV}$ without affecting the $2.22 \mathrm{MeV}$ and annihilation line emission Share et al 2004.

Then the momentum delivered by such a proton beam to the chromosphere where the $\gamma$-emission is measured, can be about $\sim 2.2 \cdot 10^{22} \mathrm{~g} \cdot \mathrm{cm} / \mathrm{s}$ for the lower energy of $30 \mathrm{MeV}$ and according to equations (12) and (14), increases as $(2 \mathrm{MeV} / 30 \mathrm{MeV})^{-\gamma+0.5} \times \frac{\gamma}{\gamma+0.5}$ to $\sim 5.2 \cdot 10^{24} \mathrm{~g} \cdot \mathrm{cm} / \mathrm{s}$ for the lower energy of $2.0 \mathrm{MeV}$ and $\gamma=3.0$. This range superbly covers those momenta derived in the sources S2 and S3 (Table 2). Hence, protons can be the agents in these two sources delivering the sufficient momenta to the region where MDI measures the Doppler velocities.

\subsection{Simulated hydrodynamic responses}

We simulate the hydrodynamics of a two-temperature plasma heated by either electrons or protons (power laws plus those with Maxwellian energy distributions) by solving the two energy equations (for electrons and protons), continuity and momentum conservation equations and include the radiative cooling from lines and continua. The heating functions 

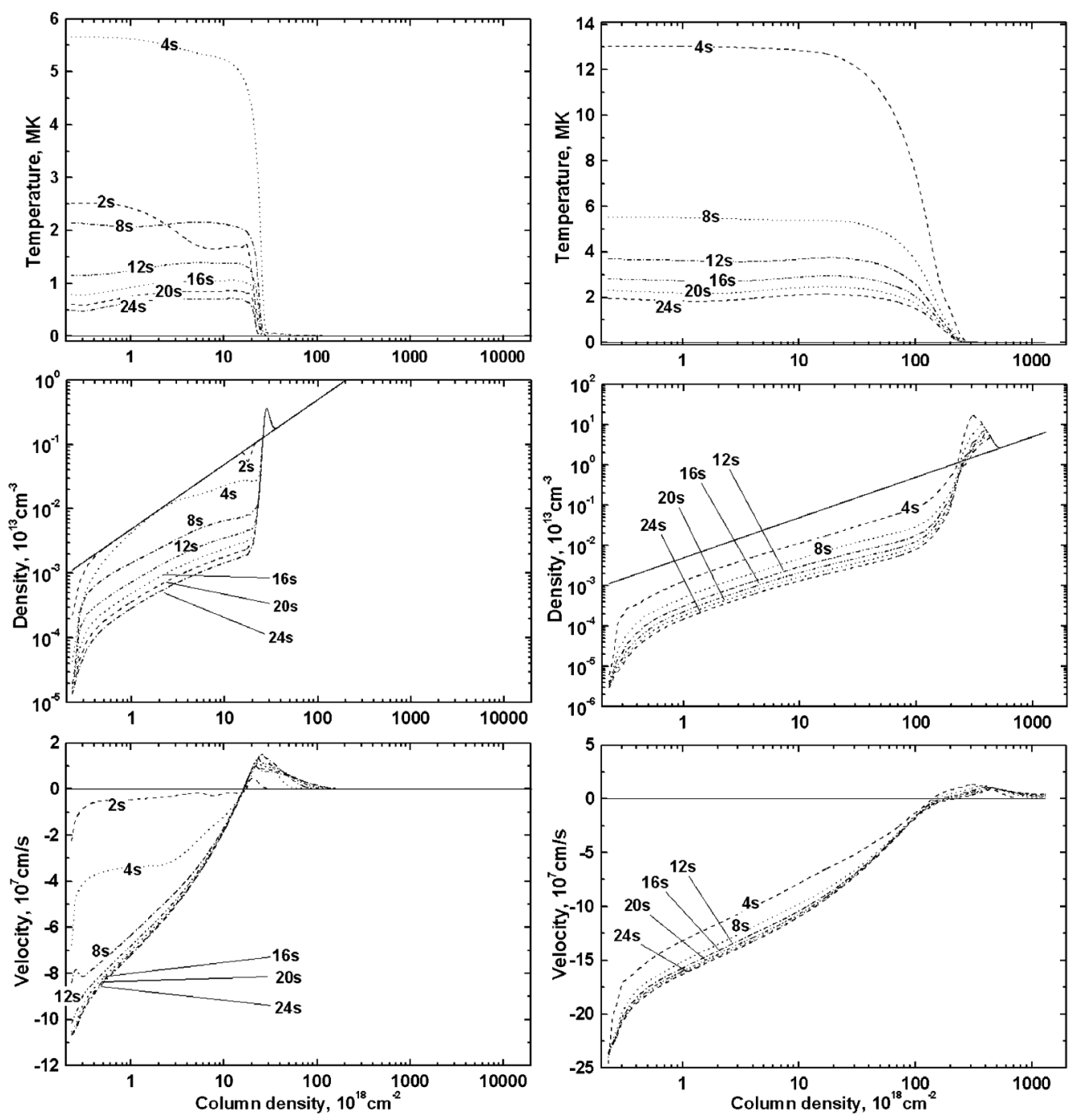

Figure 2. The hydrodynamic responses of a flaring atmosphere caused by pure electrons (the left column) or by mixed proton and electron beams (the right column) with the parameters of protons deduced from $\gamma$-rays and electrons from X-rays from RHESSI and KORONAS. The upper plots present the ambient electron temperatures, the middle ones - densities and the lower plots - macro-velocities, the numbers on the graphs show the times in seconds after the beam injection.

are considered for collisional losses by both electron and proton beams, Ohmic losses only for an electron beam and Cherenkov resonance for thermal-like protons.

The variations of temperature, density and macro-velocity simulated for the hydrodynamic responses are plotted for the electron beam with the parameters derived from hard X-rays (Figure 2, the left plots) or for the mixed proton/jet beam with the parameters derived from the $\gamma$-ray emission (Figure 2, the right plots) that agree with previous HD simulations (Somov et al., 1981); Nagai and Emslie, 1984 and Fisher et al., 1985).

Starting from the column density $2 \times 10^{19} \mathrm{~cm}^{2}$, a collisional stopping depth for lower energy electrons of $12 \mathrm{keV}$ accepted in our simulations, a low temperature condensation is formed moving as a shock with velocities of about $100-200 \mathrm{~km} / \mathrm{s}$, which are higher 
than the local sound velocity (Somov et al., 1981, Fisher et al., 1985). However, such a shock produced even by a powerful electron beam with the spectral index of about 3 (the lower plots) appears rather high in the upper chromosphere between the column depths of $2 \times 10^{19} \div 2 \times 10^{20} \mathrm{~cm}^{-2}$ as can be seen in Figure 2 (the left plots).

As can be seen from Figure 2, the hydrodynamic response to the injection of the pure electron or mixed proton/jet beams are substantially different. The electron beam injected during $10 \mathrm{~s}$ produces a smaller (by factor 2-2.5) temperature increase in the corona (top left plots), a smaller (by an order of magnitude) density depression of the coronal plasma into the chromosphere (the middle left plots) and smaller (by factor 22.5) evaporation velocities (the bottom left plots) compared to those by mixed proton/jet beams (the right plots in Figure 2).

In addition, the mixed proton/jet beam forms the lower temperature shock, which is spread much deeper into the lower chromosphere between the column depths of $2 \times$ $10^{20} \div 8 \times 10^{21} \mathrm{~cm}^{-2}$. The velocities of the shock induced by the mixed proton beam are also higher (by a factor of 2-2.5) than those induced by the pure electron beam. These macro-velocities induced by the proton beam decrease in the region with a column density of $5 \times 10^{21} \mathrm{~cm}^{-2}$ to a few $\mathrm{km} / \mathrm{s}$ compared to those less than $1 \mathrm{~km} / \mathrm{s}$ for the one induced by electrons.

The momentum induced by this shock is transferred to the photosphere within much shorter timescale under 1 minute because it is formed in much deeper and denser atmospheric levels. The proton induced shock deposits its momentum from the depths $5-8 \times 10^{21} \mathrm{~cm}^{-2}$ to very dense plasma beneath that is delievered with a velocity of about $2 \mathrm{~km} / \mathrm{s}$ through about $120 \mathrm{~km}$ of the solar atmosphere before approaching the column depth of $4 \times 10^{23} \mathrm{~cm}^{-2}$ for Ni line region. While the electron-induced shock is required to travel from the column depth of $(2-5) \times 10^{20} \mathrm{~cm}^{-2}$, or about $350 \mathrm{~km}$, with the same or the twice lower velocity that will allow the momentum to reach the Ni region with a delay of 3-6 minutes.

\subsection{The proposed scenarios}

The shock caused by a pure electron beam (Figure 2, left plots) with the beam parameters taken from KORONAS has a density about $5 \times 10^{12} \mathrm{~cm}^{-3}$, an average macro-velocity about $1.8 \times 10^{7} \mathrm{~cm} / \mathrm{s}$ and a duration about $100 \mathrm{~s}$. This shock is formed at the depth of about $(2-5) \times 10^{20} \mathrm{~cm}^{-2}$. The momentum still requires a timescale $>180 \mathrm{~s}$ to be delivered to the Ni line formation region $(2-6) \times 10^{23} \mathrm{~cm}^{-2}$. Then for the source S1 with the area of about $5.05 \times 10^{17} \mathrm{~cm}^{2}$ the electron-formed shock can deliver a momentum of about $1.3 \cdot 10^{19} \mathrm{~g} \cdot \mathrm{cm} / \mathrm{s}$.

Evidently, the shock produced by a pure electron beam does not contain enough momentum to account for the seismic responses recorded in either source. Also a time delay of about 180-360 s is required for this shock to reach the region of the Ni line formation to cause a delay in the seismic response appearance compared to the emission in hard $\mathrm{X}$-rays and $\gamma$-rays.

On the contrary, the parameters of the proton-formed shock are much more relevant to the explanation of the momenta observed in the seismic sources S1, S2 and S3. This shock occurs much closer to the region where the Ni line is formed, it contains much denser material (up to $10^{14} \mathrm{~cm}^{-3}$ ) and higher macro-velocities $\left((2-3) \times 10^{7} \mathrm{~cm} / \mathrm{s}\right.$. By substituting these parameters and the areas of the sources S1, S2 and S3 taken from Table 2 into the formula (18), one can find that this shock can deliver the much higher $\left(>4.4 \times 10^{22} \mathrm{gcm} / \mathrm{s}\right)$ momentum compared to that delivered by the electron-formed one. 
The momentum induced by this shock is transferred to the photosphere within much shorter timescale under 1 minute because it is formed in much deeper and denser atmospheric levels. Therefore, the magnitudes of the momentum carried by the shock caused by the mixed proton/jet beam and the timescale, within which it reaches the region of $\mathrm{Ni}$ line, are rather close to those deduced from the TD-diagrams.

Since $\gamma$-ray emission by high energy protons was observed by KORONAS only after 11:06:00 UT (Kuznetsov et al., 2006), when the sources S2 and S3 appeared, one can assume that these sources are produced by a high energy proton beam combined with the lower energy $(<200 \mathrm{MeV})$ quasi-thermal protons of the separatrix jets. They together can deliver to the photosphere the momenta via the hydrodynamic shock, to cause directly the observed seismic responses and to energise ambient electrons to energies high enough for the hard X-ray and $\gamma$-ray emission observed at 11:06:00 UT in the third burst.

For the source S1 sufficient momentum can be delivered by a very high energy electron beam, which is reported to have energies of hundreds of MeVs (Kuznetsov et al., 2006) and occurred at 11:02:00 UT in the first burst combined with the lower energy $(<200 \mathrm{MeV})$ quasi-thermal jet protons. Then the hydrodynamic response to such heating could lead to a shock formed slightly deeper in the flaring atmosphere than those for the electron beam from Figure 2 and slightly higher than for the protons. Within 3-4 minutes after the beam onset the momentum caused by this hydrodynamic shock can reach the Ni region and cause the observed seismic waves hich explains the delay of about 3-4 minutes between the seismic response in the source 1 and the hard X-ray emission in the first burst.

\section{References}

Donea, A.C. \& Lindsey C., 2005, Astrophys. J, 630/2, 1168.

Fisher, G.H., Canfield, R.C. \& McClymont, A.N., 1985, Astrophys. J, 289, 414.

Hurford, G., Krucker, S., Lin, R.p., Schwartz, R.A., Share, G.H. \& Smith, D.M., 2006, Astrophys. $\mathrm{J}, 644, \mathrm{~L} 93$.

Gordovskyy, M., 2005, PhD thesis., University of Bradford.

Gordovskyy, M., Zharkova, V.V., Voitenko, M.Yu. \& Goossens M., 2005, Adv. Space Res., 35, $\mathrm{N} 10,1743$.

Gross, M., Tatishchev, V., Kiener., J., Cordier, B. \& other 7 authors, 2004, Proc. 5th INTEGRAL Workshop on the INTEGRAL Universe, ESA SP-552, 16-20 February 2004, Munich, Germany, Scientific Editors: V. Schnfelder, G. Lichti \& C. Winkler, p.669.

Kiener., J., Gros, M., Tatishchev, V. \& Weidenspointner, G., 2006, A \& A, 445, 725.

Kosovichev A.G., 2006, Solar Phys., 238, 1.

Kosovichev A.G. \& Zharkova, V.V., 1998, Nature, 393, 317.

Kuznetsov, S.N., Kurt, V.G., Myagkova, I.N., Yushkov, B.Yu. \& Kudela K., 2006, Solar System Research, bf 40, N2, 104.

Nagai, F. \& Emslie, A.G., 1984, Astrophys. J, 279, 896.

Share, G., Murphy, R.J., Smith, D.M., Schwartz, R.A. \& Lin, R.P., 2004, Astrophys. J, 615, L169-L172.

Somov, B.V.; Spektor, A.R. \& Syrovatskii, S.I., 1981, Solar Phys., 73, 145.

Zharkova, V.V. \& Gordovskyy M., 2004, Astrophys.J, 604, 884.

Zharkova, V.V. \& Gordovskyy M., 2005a, MNRAS, 356, 1107.

Zharkova, V.V. \& Gordovskyy M., 2006, Astrophys.J, 651, 553. 